Rob J. De Boer · José A. M. Borghans •

Michiel van Boven - Can Keşmir · Franz J. Weissing

\title{
Heterozygote advantage fails to explain the high degree of polymorphism of the MHC
}

Received: 6 June 2003 / Revised: 11 November 2003 / Published online: 13 January 2004

(C) Springer-Verlag 2004

\begin{abstract}
Major histocompatibility (MHC) molecules are encoded by extremely polymorphic genes and play a crucial role in vertebrate immunity. Natural selection favors MHC heterozygous hosts because individuals heterozygous at the MHC can present a larger diversity of peptides from infectious pathogens than homozygous individuals. Whether or not heterozygote advantage is sufficient to account for a high degree of polymorphism is controversial, however. Using mathematical models we studied the degree of MHC polymorphism arising when heterozygote advantage is the only selection pressure. We argue that existing models are misleading in that the fitness of heterozygotes is not related to the MHC alleles they harbor. To correct for this, we have developed novel models in which the genotypic fitness of a host directly reflects the fitness contributions of its MHC alleles. The mathematical analysis suggests that a high degree of polymorphism can only be accounted for if the different MHC alleles confer unrealistically similar fitnesses. This conclusion was confirmed by stochastic simulations, including mutation, genetic drift, and a finite population
\end{abstract}

R. J. De Boer $(\bullet) \cdot$ C. Keşmir

Theoretical Biology,

Utrecht University,

Padualaan 8, $3584 \mathrm{CH}$ Utrecht, The Netherlands

e-mail: R.J.DeBoer@bio.uu.nl.

Tel.: +31-30-2537560

Fax: $+31-30-2513655$

J. A. M. Borghans

Biologie des Populations Lymphocytaires,

Institut Pasteur,

25-28 Rue du Dr Roux, 75015 Paris, France

M. van Boven

Animal Sciences Group,

Wageningen University and Research Centre,

P.O. Box 65, 8200 AB Lelystad, The Netherlands

F. J. Weissing

Theoretical Biology,

University of Groningen,

Kerklaan 30, 9750 NN Haren, The Netherlands size. Heterozygote advantage on its own is insufficient to explain the high population diversity of the MHC.

Keywords Heterozygote advantage $\cdot$ MHC polymorphism

Electronic Supplementary Material Supplementary material is available in the online version of this article at http://dx.doi.org/10.1007/s00251-003-0629-y

\section{Introduction}

The class I and class II genes from the major histocompatibility complex (MHC) encode molecules expressed on the cell surface that present short peptides to T lymphocytes. The MHC is extremely polymorphic. For the classical MHC loci, hundreds of different alleles have been identified (Parham and Ohta 1996; Vogel et al. 1999). The high rate of nonsynonymous substitutions within the MHC peptide-binding regions, compared with normal rates in other regions of the MHC, suggests that the MHC polymorphism is due to Darwinian selection rather than to genetic drift and mutation (Hughes and Nei 1988, 1989; Parham et al. 1989a, 1989b; Takahata et al. 1992). MHC molecules have different peptide-binding motifs and bind a small fraction (i.e., less than 1\%) of all peptides (Kast et al. 1994; Barouch et al. 1995; Rammensee et al. 1999). As a consequence, different MHC molecules bind largely non-overlapping sets of peptides, and hosts heterozygous for the MHC can present up to twice as many peptides from infectious pathogens as homozygous hosts.

Heterozygote advantage is the simplest mechanism explaining MHC polymorphism (Doherty and Zinkernagel 1975; Hughes and Nei 1988, 1989, 1992; Takahata and Nei 1990; Hughes and Yeager 1998; Jeffery and Bangham 2000). Classical papers in population genetics theory have suggested that heterozygote advantage is sufficient to account for the polymorphism of hundreds of 
alleles observed at various MHC loci (Maruyama and Nei 1981; Takahata and Nei 1990; Hughes and Yeager 1998). Poor alleles can persist at low frequencies because they are then infrequently expressed as homozygotes [which is a trivial rare allele effect (Apanius et al. 1997)]. The evolutionary significance of heterozygote advantage is confirmed by the fact that heterozygotes are better protected against infectious disease. For instance, MHC heterozygosity correlates negatively with disease in HIVinfected patients (Carrington et al. 1999), with HTLV-1 infection in humans (Jeffery et al. 2000), and with infectious diseases in California sea lions (AcevedoWhitehouse et al. 2003). In mouse populations infected under experimental conditions with several Salmonella strains and Listeria, MHC heterozygotes were more resistant to infection than homozygotes (Penn et al. 2002).

Although heterozygote advantage is clearly an important selection pressure, several authors have argued that an additional frequency-dependent selection pressure is required (Snell 1968; Bodmer 1972; Beck 1984; Potts et al. 1991; Slade and McCallum 1992; Apanius et al. 1997; Reusch et al. 2001; Hedrick 2002). It remains controversial, therefore, whether or not heterozygote advantage alone is sufficient to account for the observed high degree of polymorphism of the MHC. To address this question, we developed a novel population genetical model. In order to focus on the contribution of heterozygote advantage to MHC polymorphism, the model deliberately neglects a number of confounding factors. Reflecting the mechanisms underlying immune protection, we assume that the fitness of an individual is directly related to the properties of MHC alleles harbored by this individual. This is in contrast to earlier models, where the fitness of heterozygotes is supposed to be unrelated to the specificity of their MHC alleles (Maruyama and Nei 1981; Lewontin et al. 1978; Takahata and Nei 1990). We argue that the high degree of polymorphism predicted by these earlier models is directly related to this unrealistic assumption. Our results suggest that heterozygote advantage can only account for a high degree of MHC polymorphism if the fitness contributions of the alleles are unrealistically similar. In the companion paper (Borghans et al., DOI 10.1007/s00251-003-0630-5) we add host-pathogen co-evolution and show that such a form of frequency-dependent selection naturally accounts for the observed high degree of MHC polymorphism.

\section{Results}

Equilibrium results

To investigate how the number of MHC alleles maintained at equilibrium depends upon the fitness of the host genotype, we have developed a population genetical model. Formally, the model structure is identical to the standard model for selection at an autosomal locus (see, e.g., Nagylaki 1992, pp 47-51). In the model, an allele $i$ makes a fixed predefined contribution, $f_{i}$, to the overall genotypic fitness. Intuitively, the contribution $f_{i}$ corresponds to the fraction of all dangerous pathogens to which the allele can provide protection. This interpretation implies that $0 \leq f_{i} \leq 1$. Setting $f_{i}=0$ means that the allele does not recognize any pathogen, while the maximum $f_{i}=1$ means that the allele fully protects the host to all pathogens. To construct the fitness of a host's genotype from the fitness contributions of its two alleles we have to consider the overlap in the pathogens recognized by the alleles. Since the fitness contributions, $f_{i}$, are fractions the fitness of a heterozygote genotype expressing alleles $i$ and $j$ equals $f_{i j}=f_{i}+\left(1-f_{i}\right) f_{j}$. The fitness of a homozygote is simply $f_{i i}=f_{i}$. These expressions are generalized by adding a basis fitness $\beta$ that is independent of the MHC, and by defining an overlap parameter $\lambda$. Setting $\lambda=1$ yields the overlap discussed above. Alternatively, one can consider a simple additive fitness model by setting $\lambda=0$.

Thus, we consider a set of $n$ alleles at one MHC locus with fitness contribution $0 \leq f_{i} \leq 1$, which are present with frequency $p_{i}$. The fitness of a homozygote is defined as $f i_{i}=\beta+f_{i}$, while the fitness of a heterozygote is defined as $f_{i j}=\beta+f_{i}+f_{j}-\lambda f_{i} f_{\mathrm{j}}$. The frequency distribution of $n$ MHC alleles at steady state follows from the requirement that the marginal fitnesses, $w_{i}=\sum_{j=1}^{n} p_{j} f_{i j}$, should be identical for all alleles $i$ (see Appendix). Persistence of allele $i$ at equilibrium requires

$f_{i}>\frac{n-1}{n} \hat{f}$

where $\hat{f} \equiv n / \sum_{j=1}^{n} f_{j}^{-1}$ is the harmonic mean of the $n$ allelic fitness contributions. This shows that the number of alleles persisting at equilibrium is independent of the choice of $\lambda$, and that the basis fitness value $\beta$ has canceled (Weissing and Van Boven 2001). Importantly, Eq. 1 implies that coexistence of many alleles requires that their fitness contributions, $f_{i}$, have to be very close to one another (i.e., for large $n$ the ratio $(n-1) / n$ goes to 1$)$. For example, 20 alleles can only coexist at equilibrium if the fitness contribution of each allele exceeds 0.95-times the harmonic mean of the fitness contributions of all 20 alleles. Hence, already the smallest of the fitness contributions has to be close to the harmonic mean, implying that the fitness contributions cannot differ from another too much. Additionally, Eq. 1 shows that the critical fitness contribution increases with the degree of polymorphism $n$. Thus, the larger the polymorphism one has to explain with a model like this, the more similar the required fitness contributions of the alleles.

One can also compute the conditions for the successful invasion of novel alleles into this steady state (Weissing and Van Boven 2001; Van Boven and Weissing 2001). In the Appendix, we derive that a novel, i.e., the $n+1^{\text {th }}$, allele will invade into a steady state polymorphism of $n$ established alleles whenever it has a fitness contribution of

$f_{n+1}>\frac{n-1}{n} \hat{f}$ 
which is similar to Eq. 1. Thus, for a set of alleles that are ranked in their fitness contribution $f_{i}$ (such that $f_{1}>\ldots>f_{n}$ ), novel alleles will only be able to invade if their fitness contribution is sufficiently close to the harmonic mean fitness contribution $\hat{f}$ of the $n$ established alleles. We studied a specific example with $f_{i}=(1-s)^{i-1}$, where $s$ defines the "steepness" with which the fitness contribution decreases with the allele number. Solving $s$ from Eq. 2 gives the maximum $s$ value that is compatible with a polymorphism of $n+1$ alleles (see Appendix). The larger the polymorphism, the smaller the variation in allelic fitness contributions, i.e., the smaller $s$ (see Fig. 1a). Similarly, the larger the degree of polymorphism, the higher the critical fitness contribution of an invading allele (see Fig. 1a). Figure 1b depicts the corresponding frequency distributions of the persisting alleles for various values of the steepness parameter $s$. Summarizing, heterozygote advantage can only account for a high degree of MHC polymorphism if the variation in fitness contributions amongst the MHC alleles is vanishingly small.

To check our analysis for arbitrary fitness contributions of the alleles, we randomly drew large numbers of $f_{i}$ values from a uniform distribution, $0<f_{i}<1$, and solved the standard model for viability selection at an autosomal locus [see Nagylaki (1992), pp 47-51] until the steady state was approached. This confirmed the above results, as the fitness contributions of the small set of surviving alleles were unrealistically similar (not shown). Also note that, although it is difficult to explain a high degree of polymorphism with this model, the model easily accounts for a low degree of polymorphism by heterozygote advantage. According to Eq. 2, a second allele can always invade a monomorphic population $(n=1)$, whatever the fitness contribution, $f_{2}$, of the novel allele.

\section{Simulation results}

The model analyzed above can at best be an approximation to the real world, since it assumes an infinite population size, thereby neglecting processes like mutation and genetic drift. To investigate whether our analysis also applies to more realistic circumstances, we developed a stochastic simulation model in which MHC alleles arise by mutation, and in which the host population size is finite. The simulation model is again allele based: individual hosts inherit MHC alleles from both parents, and the host genotypic fitness depends on the fitness contributions of both alleles. Mutation occurs at birth with a probability of $10^{-5}$. A mutation creates a new allele by randomly drawing a fitness contribution $0<f_{i}<1$ from a uniform distribution.

Because we here consider immune protection conferred by MHC molecules, it seems most realistic to assume that the fitness of an individual determines its expected life-span. For instance, homozygous hosts tend to have a shorter expected life-span, and therefore have a smaller lifetime reproductive success. To study an evolv-

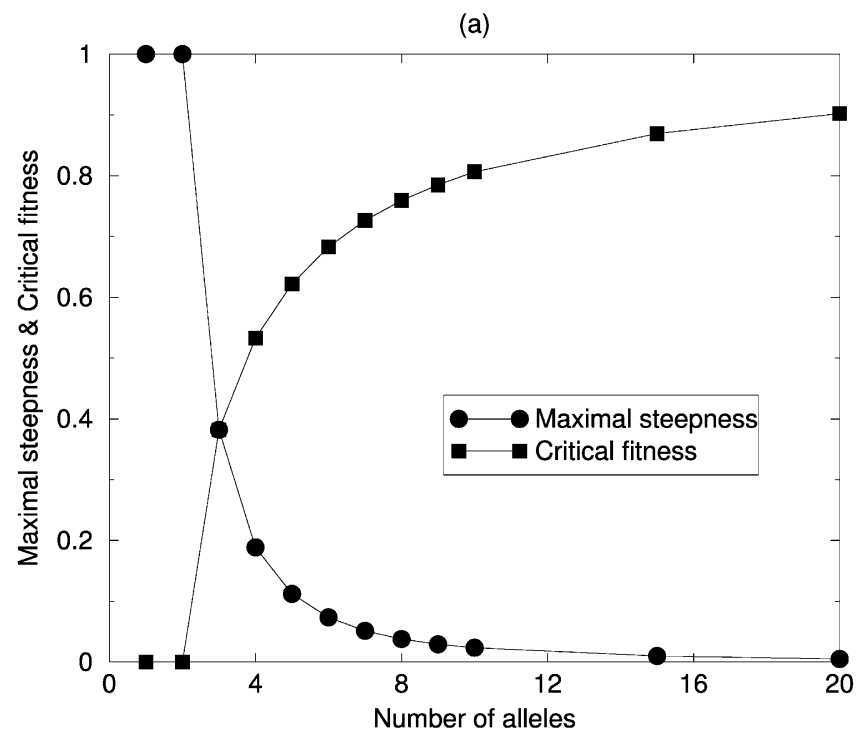

(b)

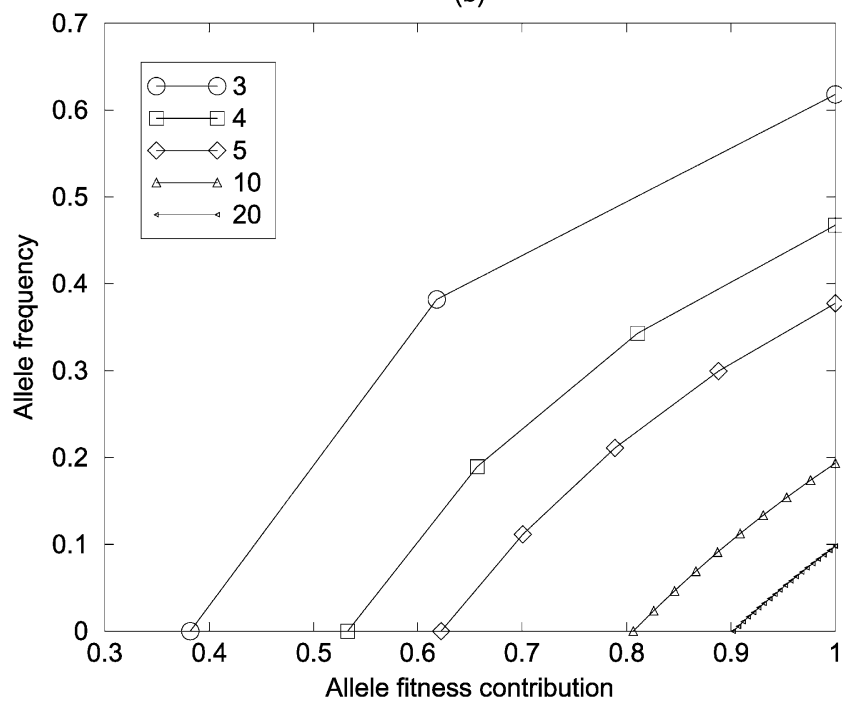

Fig. 1a, b The MHC polymorphism obtained at equilibrium when the allelic fitness contributions are of the form $f_{i}=(1-s)^{i-1}$. Panel a depicts the maximal value of $s$ allowing the coexistence of a given number of alleles (circles) and the minimal fitness required for a new allele for successful invasion in the resulting equilibrium population (squares). The latter approaches the harmonic mean fitness $\hat{f}$ when $n$ grows large. Panel $\mathbf{b}$ depicts the corresponding distributions of allele frequencies for $n+1=3,4,5,10$ and 20 alleles, i.e., for $s=0.38,0.19,0.11,0.02$ and 0.005 , respectively

ing human population, individuals with maximum fitness $f_{i j}=1$ were given an expected life-span of 50 years. For lower fitness values the expected life-span was proportional to the host genotypic fitness, which - like above - was determined by the fitness contribution of the alleles. Thus, for homozygotes we set $f_{i i}=f_{i}$, and the genotypic fitness of a heterozygous host was $f_{i j}=f_{i}+f_{j}-f_{i} f_{j}$ (i.e., we consider $\lambda=1$ ). Because hosts have different expected life-spans, they have overlapping generations, which is realistic for the human population that we are simulating. Simulations were started with 100 alleles with 
fitness contributions $0<f_{i}<1$ that were drawn from a uniform distribution and were distributed randomly over the fixed population size of 10,000 individual hosts. The individual mortality was set to $1-0.98 f_{i j}$ per time-step to allow for a life expectancy of 50 time-steps (years) when $f_{i j}=1$. Each individual that died was replaced by a new individual whose two MHC alleles were drawn from two randomly chosen parents. The simulation was coded in the C-programming language and is available as Electronic Supplementary Material in the online version of this article at http://dx.doi.org/10.1007/s00251003-0629-y

A typical example of such a stochastic simulation of our "allele based" model is shown in Fig. 2a. The number of alleles very rapidly drops from the initial 100 to about ten, and fluctuates around a polymorphism of approximately ten alleles for another million years. The small spikes in the curve represent mutation events. Some of the mutants will have a high enough fitness contribution $f_{i}$, and will invade into the population. Apparently this drives earlier alleles to extinction; otherwise the degree of polymorphism would continuously increase. The low degree of polymorphism approached in these simulations is in good agreement with the results of the equilibrium model. The frequency distribution of the alleles present in the population after a million years is depicted in Fig. 2c. This also confirms the results of the equilibrium model: the persisting alleles have high and very similar fitness contributions.

Our results differ remarkably from those of the classical study of Takahata and Nei (1990). Based on a stochastic model that seems to resemble that of the present study, they predicted a much higher degree of polymorphism (Maruyama and Nei 1981; Takahata and Nei 1990). What causes the discrepancy in the predictions of the two models? In their model, Takahata and Nei (1990) chose the fitness of homozygotes at random and set the fitness of heterozygotes to one. Hence, the fitness of a heterozygote individual does not reflect the MHC alleles harbored by this individual. It is not surprising that a model with constant and high heterozygote fitness values does produce a high degree of polymorphism. In fact, in such a model any allele will have a high marginal fitness whenever it is rare (namely the fitness of heterozygote individuals) and, hence, will be able to persist. In the Appendix, we present a formal proof of this fact: in the absence of genetic drift, alleles can never go extinct if all heterozygotes have the same maximal fitness.

To confirm these general considerations, we repeated our simulations, but now reset the fitness of heterozygotes to $f_{i j}=1$. Indeed, these simulations yielded a much higher degree of polymorphism than our allele-based model (see Fig. 2a, b). We conclude that the large degree of MHC polymorphism obtained in the Takahata and Nei (1990) model was a consequence of the unrealistic assumption that all heterozygotes have the same fitness. Interestingly, the alleles persisting after one million time-steps in our allele-based simulations (Fig. 2c) come very close to satisfying the assumptions of the Takahata and Nei (1990) (a)

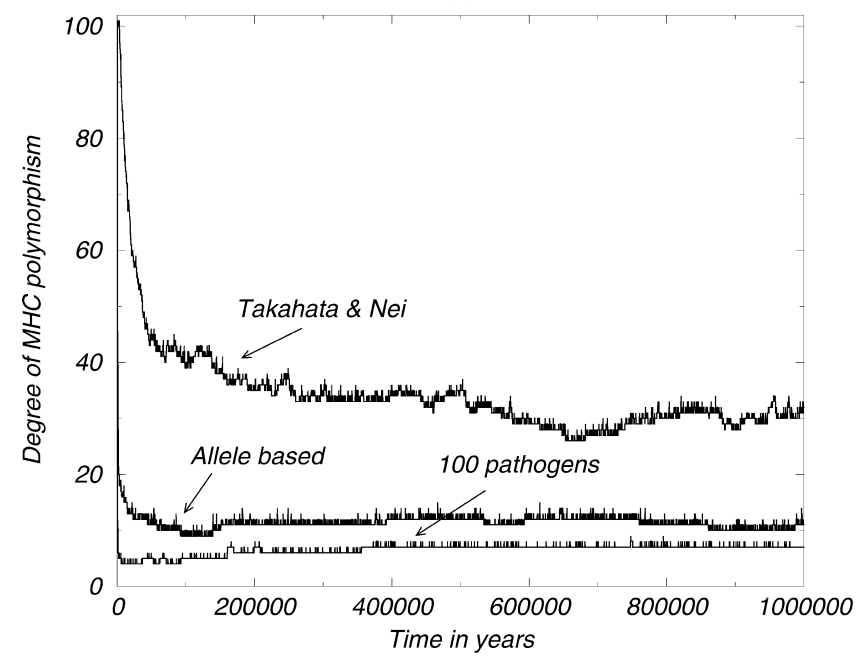

(b)

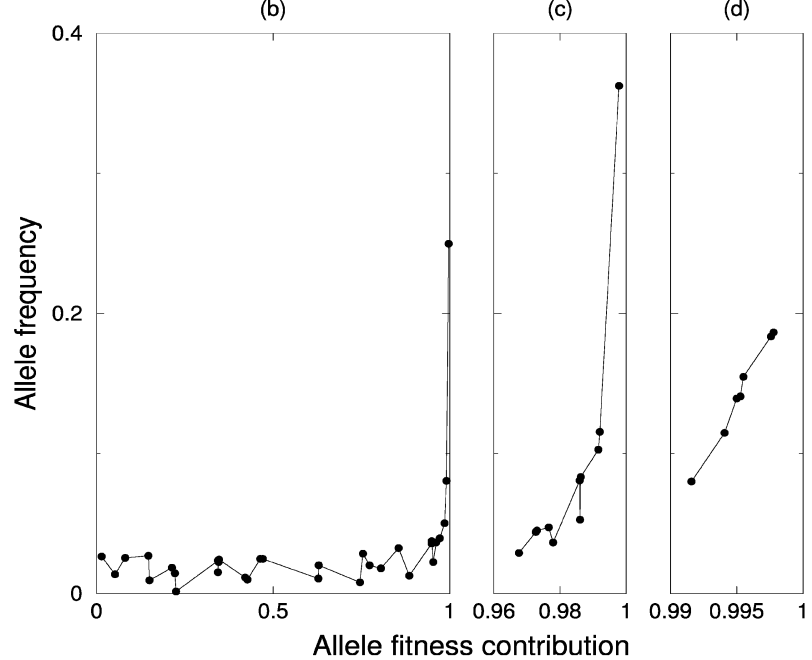

Fig. 2a-d MHC polymorphism evolving in stochastic simulations under varying model assumptions. Panel $\mathbf{a}$ is a time plot of the number of alleles evolving in three different models, while panels b-d show for each of the models the frequency distribution of persisting alleles. Panel b corresponds to the Takahata and Nei (1990) model, where the fitness of all heterozygotes is equal to one. Panel c depicts the results of our allele-based model (for $\lambda=1)$. Panel d illustrates the " 100 pathogens" extension of the allele based model, that was made by setting $f_{i i}=f_{i}^{100}$ and $f_{i j}=\left(f_{i}+f_{j}-f_{i} f_{j}\right)^{100}$. The figure shows one representative simulation; all simulations performed produced similar results

model. In line with the equilibrium analysis above, all persisting alleles had very similar fitness contributions, i.e., $f_{i}>0.965$. As a consequence, even the lowest heterozygote fitness was larger than $f_{i j}=0.999$, a value that is very close to the $f_{i j}=1$ assumption of Takahata and Nei (1990). This, however, only holds for the persisting alleles. The other alleles are not able to persist just because they induce a heterozygote fitness that is considerably smaller than one.

Lewontin and co-workers (1978) constructed fully random genotypic fitness matrices, and concluded that a high degree of polymorphism is only possible if the 
fitness difference between homozygotes and heterozygotes is sufficiently large. In our model, in which the fitness contribution of an allele represents the fraction of dangerous pathogens presented by an MHC allele, the maximum fitness difference between heterozygotes and homozygotes is twofold. To test if larger differences between the fitnesses of heterozygotes and homozygotes in our allele-based model could give rise to higher degrees of MHC polymorphism, we re-interpreted the fitness contribution $f_{i}$ as the probability that an MHC allele provides protection to a single pathogen. Defining the genotypic fitness as the likelihood of surviving $k$ pathogens, the fitness difference between heterozygotes and homozygotes becomes larger than twofold and increases with $k$ (see the legend of Fig. 2 for the formula). Stochastic simulations with $k=100$ pathogens lowered the degree of MHC polymorphism (see Fig. 2a, d). This is also in good agreement with the results of the deterministic model of Eq. 2, because the fitness difference between high-ranking MHC alleles, i.e., $f_{i} \simeq 1$, becomes larger when $k$ is increased. Thus, in an allele-based approach, a large difference between the fitnesses of heterozygotes and homozygotes is insufficient to obtain a high degree of MHC polymorphism: one still requires that the fitness contributions of the alleles are exceedingly similar.

\section{Discussion}

The evolution of polymorphism at the MHC is under the influence of many different selection pressures, and heterozygote advantage is only one of them. Our results establish one simple but important new insight: if heterozygote advantage were to be the only selection pressure, it could only explain the observed high degree of MHC polymorphism if the fitness contributions of the alleles would be exceedingly similar. This result was established with the analytical model of Eq. 2, and was subsequently confirmed with a more realistic simulation model [see also the companion paper (Borghans et al., DOI 10.1007/s00251-003-0630-5)]. The maximum degree of MHC polymorphism that heterozygote advantage in these simulation models can typically account for is about ten alleles, and even this degree of polymorphism requires that the fitness contribution of the worst allele in the population is relatively high. Thus, to explain observed polymorphisms greatly exceeding ten alleles, one requires at least one of the additional selection pressures, e.g., the host-pathogen co-evolution considered in the companion paper (Borghans et al., DOI 10.1007/s00251003-0630-5).

One could argue that evolution leads to a slow accumulation of novel MHC alleles with very similar fitness contributions (Lewontin et al. 1978). We think this is not the case because even MHC alleles from the same supertype differ greatly in their binding motifs (Barouch et al. 1995; Sudo et al. 1995; Rammensee et al. 1999), and small binding-motif differences can lead to large differ- ences in protection. For instance, a one-amino-acid difference in the peptide-binding region of the $D R B 1 * 1302$ allele abrogates its protection to malaria (Davenport et al. 1995). Protective HLA alleles have been described for a number of infectious diseases (Van Eden et al. 1980; Hill et al. 1991; Klein et al. 1994), and the associated fitness differences are much larger than the small differences required for explaining the high degree of HLA polymorphism. The fact that the HLA alleles expressed in the South Amerindian population are different from those in the founder population, while North Amerindians still express the founder alleles (Parham and Ohta 1996), also suggests that different MHC alleles provide different degrees of protection against different pathogens. Since immunity against dangerous viruses can be a matter of life and death, small differences in antigen presentation between MHC alleles can lead to large host fitness differences, which suggests that the fitness contributions of MHC alleles in the human population are not sufficiently similar to explain the high degree of MHC polymorphism by heterozygote advantage selection only. Remember that even a low polymorphism of $20 \mathrm{MHC}$ alleles requires that the fitness contribution of the worst allele is at least $95 \%$ of the harmonic mean of the fitness contributions of the other 19 alleles (see above).

To study MHC evolution, our more mechanistic allelebased modeling approach seems a major improvement over the previous models. Our computer simulations revealed large differences between models drawing random uncorrelated genotypic fitnesses (Lewontin et al. 1978; Maruyama and Nei 1981; Takahata and Nei 1990) and models drawing random uncorrelated fitness contributions of alleles leading to correlated genotypic fitnesses. Since MHC alleles tend to have quite different binding motifs, one does expect correlations between genotypes expressing the same MHC alleles, and we have shown that these correlations influence the degree of MHC polymorphism that one expects to evolve under heterozygote advantage selection.

From a biological point of view one could still argue that the fitness contributions of the various MHC alleles are sufficiently similar to account for a high degree of polymorphism. It is now well established that different MHC alleles provide different levels of protection to specific pathogens (Van Eden et al. 1980; Hill et al. 1991; Klein et al. 1994). One could nevertheless argue that by averaging over many different pathogens the lifetime fitness contributions of MHC alleles would become very similar, and hence that heterozygote advantage would be able to account for a high degree of MHC polymorphism. Averaging over all pathogens is not the correct procedure for obtaining a lifetime fitness contribution of an MHC allele, however. Whenever an allele has poor performance to one deadly pathogen, hosts carrying that allele would have a severe fitness cost. Thus, the minimum fitness contribution of an allele seems a better predictor of its lifetime fitness contribution than its average fitness contribution. The experimental fact that hosts heterozygous for the MHC are better protected against infectious 
disease (Carrington et al. 1999; Jeffery et al. 2000; Penn et al. 2002; Acevedo-Whitehouse et al. 2003) also argues for significant fitness differences between MHC haplotypes.

It may seem surprising that our simulation models can select for very small fitness differences (see Fig. 2). In our allele-based model the microscopic differences between the fitness contributions of the various alleles become macroscopically large because the alleles contribute to the genotypic fitness of every host in which the allele is expressed. This is not possible in models where the heterozygote fitness is not dependent on the fitness contributions of the two alleles (Lewontin et al. 1978; Maruyama and Nei 1981; Takahata and Nei 1990). Whenever the heterozygote fitness depends on the allelic fitness contributions, the marginal fitness of the alleles is obtained by summing over all hosts. The marginal fitnesses have apparently enough variation that small differences between the fitness contributions of alleles can be selected for in populations with a finite size and genetic drift. Finally, one could argue that in our simulations we have a linear dependence between the expected life-span of a host and its genotypic fitness. Indeed, if we had incorporated saturation effects, rather than a linear dependence, the fitness contributions of alleles to the expected life-spans would have become more similar. The same simulation model would then account for a larger degree of polymorphism. Basically, this remains in good agreement with our main result: one obtains a high degree of polymorphism if, and only if, the fitness contributions of MHC alleles are very similar. Allowing for saturation effects is a means to make alleles more similar. However the fact that heterozygotes are better protected against infectious disease (Carrington et al. 1999; Jeffery et al. 2000; Penn et al. 2002; AcevedoWhitehouse et al. 2003) strongly suggests that the fitness contributions of MHC haplotypes are not similar enough to explain a high degree of MHC polymorphism by heterozygote advantage only.

Acknowledgements We thank Ludo Pagie for critically reading the manuscript. J.B. was supported by a Marie Curie Fellowship of the EC program Quality of Life (contract 1999-01548) and C.K. by a fellowship from the Bioinformatics Program of the Dutch Science Foundation (NWO PGBMI 015).

\section{Appendix}

Standard population genetical theory (Nagylaki 1992) implies that all alleles $i$ present at equilibrium need to have the same marginal fitness, $w_{i}=\sum_{j=1}^{n} p_{j} f_{i j}$, where $p_{i}$ is the frequency of allele $i$, and $f_{i j}$ is the genotypic fitness. From this condition one obtains $f_{i}\left(1-\left(1-\lambda f_{i}\right) p_{i}-\lambda \bar{f}\right)=f_{j}\left(1-\left(1-\lambda f_{j}\right) p_{j}-\lambda \bar{f}\right)$ for all alleles $i$ and $j$. Here, $\bar{f}=\sum_{k=1}^{n} p_{k} f_{k}$ is the weighted mean allelic fitness contribution. By means of the last identity, one can express all equilibrium frequencies $p_{j}$ by the same $p_{i}$. Summing up the resulting $n$ equations, one obtains $p_{i}=\frac{1-\lambda \bar{f}}{1-\lambda f_{i}}\left(1-\frac{n-1}{n} \bar{f} \bar{f}_{i}\right)$

where $\hat{f} \equiv n / \sum_{j=1}^{n} f_{j}^{-1}$ is the harmonic mean of the $n$ allelic fitness contributions. Since the leading term is strictly positive, the frequency $p_{i}$ is positive if and only if $f_{i}>\frac{n-1}{n} \hat{f}$. This shows that MHC alleles with a too low fitness contribution $f_{i}$ cannot persist at equilibrium. Note that this condition for persistence is independent of the choice of $\lambda$ and the basis fitness value $\beta$ (Weissing and Van Boven 2001; Van Boven and Weissing 2001).

For a novel allele with fitness contribution $f_{n+1}$ to invade into an established polymorphism of $n$ alleles, its marginal fitness has to exceed the marginal fitness of the other alleles (Weissing and Van Boven 2001). Writing $w_{n+1}>w_{i}$, this yields

$$
\begin{aligned}
& \sum_{j=1}^{n} p_{j}\left(f_{n+1}+f_{j}-\lambda f_{n+1} f_{j}\right)>\sum_{j=1}^{n} p_{j}\left(f_{i}+f_{j}-\lambda f_{i} f_{j}\right) \\
& \quad-p_{i} f_{i}\left(1-\lambda f_{i}\right),
\end{aligned}
$$

which can be simplified into

$f_{n+1}>f_{i}\left(1-p_{i} \frac{1-\lambda f_{i}}{1-\lambda \bar{f}}\right)$

Substituting $p_{i}$ from Eq. 5 gives Eq. 2 in the Results.

To obtain Fig. 1, we substitute $f_{i}=(1-s)^{i-1}$ into Eq. 2 (Results), where $\phi \equiv(1-s)$; one can test the invasion of the $n+1^{\text {th }}$ allele, and simplify to obtain

$\sum_{i=0}^{n}(1-s)^{i}=\frac{1-(1-s)^{n+1}}{s}>n$,

which can be used to solve the critical $s$ for invasion into any polymorphism of $n$ alleles.

In the asymmetric scenario of Takahata and Nei (1990) heterozygote genotypes all had a fitness $f_{i j}=1$, whereas the homozygote genotypic fitnesses were set to random values $f_{i i}=f_{i}$. Requiring the same marginal fitness $w_{i}=$ $\sum_{j=1}^{n} p_{j} f_{i j}$ for all alleles yields $p_{i}\left(1-f_{i}\right)=p_{j}\left(1-f_{j}\right)$ for all $j$. By the same procedure one now obtains

$p_{i}=\frac{1}{n} \frac{1-\bar{f}}{1-f_{i}}$,

which is strictly positive for $0<\bar{f}, f_{i}<1$. Since the marginal fitness of a novel invading allele is one, whereas that of the established alleles is always smaller than one, new alleles can always invade, and poor alleles will never go extinct.

\section{References}

Acevedo-Whitehouse K, Gulland F, Greig D, Amos W (2003) Inbreeding: disease susceptibility in California sea lions. Nature 422:35 
Apanius V, Penn D, Slev PR, Ruff LR, Potts WK (1997) The nature of selection on the major histocompatibility complex. Crit Rev Immunol 17:179-224

Barouch D, Friede T, Stevanovic S, Tussey L, Smith K, RowlandJones S, Braud V, McMichael A, Rammensee HG (1995) HLAA2 subtypes are functionally distinct in peptide binding and presentation. J Exp Med 182:1847-1856

Beck K (1984) Coevolution: mathematical analysis of host-parasite interactions. J Math Biol 19:63-77

Bodmer WF (1972) Evolutionary significance of the HLA system. Nature 237:139-145

Borghans JAM, Beltman JJ, De Boer RJ MHC polymorphism under hostpathogen coevolution. Immunogenetics. DOI 10.1007/ s00251-003-0630-5

Carrington M, Nelson GW, Martin MP, Kissner T, Vlahov D, Goedert JJ, Kaslow R, Buchbinder S, Hoots K, O'Brien SJ (1999) HLA and HIV-1: heterozygote advantage and B*35Cw*04 disadvantage. Science 283:1748-1752

Davenport MP, Quinn CL, Chicz RM, Green BN, Willis AC, Lane WS, Bell JI, Hill AV (1995) Naturally processed peptides from two disease-resistance-associated $H L A-D R 13$ alleles show related sequence motifs and the effects of the dimorphism at position 86 of the HLA-DR $\beta$ chain. Proc Natl Acad Sci USA 92:6567-6571

Doherty PC, Zinkernagel RM (1975) Enhanced immunological surveillance in mice heterozygous at the $\mathrm{H}-2$ gene complex. Nature 256:50-52

Hedrick PW (2002) Pathogen resistance and genetic variation at MHC loci. Evolution 56:1902-1908

Hill AV, Allsopp CE, Kwiatkowski D, Anstey NM, Twumasi P, Rowe PA, Bennett S, Brewster D, McMichael AJ, Greenwood BM (1991) Common west African HLA antigens are associated with protection from severe malaria. Nature 352:595-600

Hughes AL, Nei M (1988) Pattern of nucleotide substitution at major histocompatibility complex class I loci reveals overdominant selection. Nature 335:167-170

Hughes AL, Nei M (1989) Nucleotide substitution at major histocompatibility complex class II loci: evidence for overdominant selection. Proc Natl Acad Sci USA 86:958-962

Hughes AL, Nei M (1992) Models of host-parasite interaction and MHC polymorphism. Genetics 132:863-864

Hughes AL, Yeager M (1998) Natural selection at major histocompatibility complex loci of vertebrates. Annu Rev Genet $32: 415-435$

Jeffery KJ, Bangham CR (2000) Do infectious diseases drive MHC diversity? Microbes Infect 2:1335-1341

Jeffery KJ, Siddiqui AA, Bunce M, Lloyd AL, Vine AM, Witkover $\mathrm{AD}$, Izumo S, Usuku K, Welsh KI, Osame M, Bangham CR (2000) The influence of HLA class I alleles and heterozygosity on the outcome of human $\mathrm{T}$ cell lymphotropic virus type $i$ infection. J Immunol 165:7278-7284

Kast WM, Brandt RM, Sidney J, Drijfhout JW, Kubo RT, Grey HM, Melief CJ, Sette A (1994) Role of HLA-A motifs in identification of potential CTL epitopes in human papillomavirus type 16 E6 and E7 proteins. J Immunol 152:3904-3912

Klein MR, Keet IP, D'Amaro J, Bende RJ, Hekman A, Mesman B, Koot M, De Waal LP, Coutinho RA, Miedema F (1994) Associations between HLA frequencies and pathogenic features of human immunodeficiency virus type 1 infection in seroconverters from the Amsterdam cohort of homosexual men. J Infect Dis 169:1244-1249
Lewontin RC, Ginzburg LR, Tuljapurkar SD (1978) Heterosis as an explanation for large amounts of genic polymorphism. Genetics 88:149-170

Maruyama T, Nei M (1981) Genetic variability maintained by mutation and overdominant selection in finite populations. Genetics 98:441-459

Nagylaki T (1992) Introduction to theoretical population genetics. Springer, Berlin Heidelberg New York

Parham P, Ohta T (1996) Population biology of antigen presentation by MHC class I molecules. Science 272:67-74

Parham P, Benjamin RJ, Chen BP, Clayberger C, Ennis PD, Krensky AM, Lawlor DA, Littman DR, Norment AM, Orr HT, et al (1989a) Diversity of class I HLA molecules: functional and evolutionary interactions with T cells. Cold Spring Harbor Symp Quant Biol 54:529-543

Parham P, Lawlor DA, Lomen CE, Ennis PD (1989b) Diversity and diversification of HLAA, B,C alleles. J Immunol 142:39373950

Penn DJ, Damjanovich K, Potts WK (2002) MHC heterozygosity confers a selective advantage against multiple-strain infections. Proc Natl Acad Sci USA 99:11260-11264

Potts WK, Manning CJ, Wakeland EK (1991) Mating patterns in seminatural populations of mice influenced by MHC genotype. Nature 352:619-621

Rammensee H, Bachmann J, Emmerich NP, Bachor OA, Stevanovic S (1999) SYFPEITHI: database for MHC ligands and peptide motifs. Immunogenetics 50:213-219

Reusch TB, Haberli MA, Aeschlimann PB, Milinski M (2001) Female sticklebacks count alleles in a strategy of sexual selection explaining MHC polymorphism. Nature 414:300-302

Slade RW, McCallum HI (1992) Overdominant vs. frequencydependent selection at MHC loci. Genetics 132:861-864

Snell GD (1968) The $H-2$ locus of the mouse: observations and speculations concerning its comparative genetics and its polymorphism. Folia Biol Praha 14:335-358

Sudo T, Kamikawaji N, Kimura A, Date Y, Savoie CJ, Nakashima H, Furuichi E, Kuhara S, Sasazuki T (1995) Differences in MHC class I self peptide repertoires among HLA-A2 subtypes. J Immunol 155:4749-4756

Takahata N, Nei M (1990) Allelic genealogy under overdominant and frequency-dependent selection and polymorphism of major histocompatibility complex loci. Genetics 124:967-978

Takahata N, Satta Y, Klein J (1992) Polymorphism and balancing selection at major histocompatibility complex loci. Genetics 130:925-938

Van Boven M, Weissing FJ (2001) Competition at the mouse complex: rare alleles are inherently favored. Theor Popul Biol 60:343-358

Van Eden W, De Vries RR, Mehra NK, Vaidya MC, D'Amaro J, Van Rood JJ (1980) HLA segregation of tuberculoid leprosy: confirmation of the DR2 marker. J Infect Dis 141:693-701

Vogel TU, Evans DT, Urvater JA, O'Connor DH, Hughes AL, Watkins DI (1999) Major histocompatibility complex class I genes in primates: co-evolution with pathogens. Immunol Rev 167:327-337

Weissing FJ, Van Boven M (2001) Selection and segregation distortion in a sex-differentiated population. Theor Popul Biol $60: 327-341$ 